\title{
A professional commitment scale for clinical nurses: A study protocol
}

\author{
Fateme Jafaraghaee ${ }^{1}$, Abbas Ebadi $^{2}$, Reza Negarandeh $^{3}$, Neda Mehrdad $^{4 *}$ \\ Received: 07 Jan 2017 \\ Published: 23 Dec 2017
}

\begin{abstract}
Background: As the largest and most fundamental group targeted by programs developed to improve quality of care, nurses should be the focus of attention in the management of human resources in the field of health care. A review of literature on this subject revealed the lack of scales designed for measuring professional commitment in nurses. The present study was designed to develop a scale to measure professional commitment in clinical nurses and examine its psychometric properties.

Methods: This study will develop a professional commitment scale for clinical nurses within one qualitative and one quantitative step. The first step of the study will entail a review of the literature on the subject and interviews with clinical nurses so as to develop the categories and the items. The second step, the quantitative step, will analyze the items extracted in the qualitative step, determining the features of the topics and factor structure of the scale. The initial scale will be completed by 300 clinical nurses. The correlation among the topics, the construct validity, and reliability of the scale will also be examined in this step.

Results and Discussion: This study will develop an instrument that can measure professional commitment in clinical nurses. Given the lack of instruments for measuring professional commitment in clinical nurses, the findings of this study may be of help to nursing managers, authorities, and the health system in general.
\end{abstract}

Keywords: Protocol, Clinical nursing, Commitment

Copyright $@$ Iran University of Medical Sciences

Cite this article as: Jafaraghaee F, Ebadi A, Negarandeh R, Mehrdad N. A professional commitment scale for clinical nurses: A study protocol. Med J Islam Repub Iran. 2017 (23 Dec);31:123. https://doi.org/10.14196/mjiri.31.123

\section{Introduction}

Competent nurses are considered rare human resources in many countries (1-3). Policymakers try to solve the issue through increasing nursing training centers and the number of new nursing workforce through employing more nurses $(4,5)$. According to studies, just like many countries across the world, Iran is also short of nursing personnel $(6,7)$. Moreover, nurses working in different medical departments are unsatisfied with their job $(8,9)$. Studies show that nurses enter this profession without ample knowledge and willingness, and they plan to leave their job at least several times during their years of work $(10,11)$. If unable to carry out these plans, nurses end up limiting themselves to maintain minimum professional standards that merely help them secure their job (12). However, the desire to leave the profession has an inverse correlation with professional commitment. Nurses with higher professional commitment think less about leaving

Corresponding author:Dr Neda Mehrdad,nmehrdad@tums.ac.ir

1. Social Determinants of Health Research Center, Guilan University of Medical Sciences, Rasht, Iran.

2. Behavioral Sciences Research Center, Life Style Institute and Faculty of Nursing, Baqiyatallah University of Medical Sciences, Tehran, Iran.

3. Nursing and Midwifery Care Research Center, Tehran University of Medical Sciences, Tehran, Iran.

4. Endocrinology and Metabolism Research Center, Endocrinology and Metabolism Clinical Sciences Institute, Tehran University of Medical Sciences, Tehran, Iran. their job than their peers $(13,14)$.

Behavioral science theorists believe that professional commitment is not limited to prevent turnover; rather, it results in more valuable consequences and is considered the essence of helpful and efficient behaviors in the personnel (15). Studies suggest that professional commitment is the cause of professional competence and argue that the presence of nurses with a higher professional commitment improves the public image of nursing profession for patients and the society at large $(4,16)$. Presenting strategies that guide nurses toward achieving the professional objectives of nursing and the competent provision of nursing services are a priority in nursing. Professional commitment has been known as the main source of positive professional behavior in health care personnel and is vital to health care centers due to its correlation with the quality of patient care. The growing competition between health

$\uparrow$ What is "already known" in this topic:

Several scales exist to measure professional commitment in clinical nurses; however, none of them has been developed for Iranian nurses.

\section{$\rightarrow$ What this article adds:}

In this article, all steps to reach a new scale for measuring professional commitment in clinical nurses were presented. 
care systems for receiving greater resources directs them toward seeking ways to improve commitment among their personnel and preserve their human and financial resources (15). Professional commitment is defined as loyalty, the desire to stay in a profession, and a sense of responsibility toward the profession's particular problems and challenges. Commitment to nursing implies commitment to provide an optimal patient care and promote the nursing profession (14, 17-19).

An overview of the literature on this topic did not reveal any instruments to have been designed for measuring professional commitment in clinical nurses. Moreover, a number of studies that measured professional commitment had merely used miscellaneous instruments that measured a range of other concepts of professional commitment. Studies conducting a quantitative assessment of professional commitment have used such instruments as Blau's Career Commitment Scale (1985), Langford's Attitudinal Commitment Scale (1979), and the Health Care Professional Attitude Scale (20-22) to measure professional commitment. Nevertheless, professional commitment appears to be a special concept that needs to be measured with its own dedicated instruments.

The Organizational Commitment Questionnaire developed by Porter and Smith (1970) is one of the instruments used for measuring professional commitment that focuses on the concept of organizational commitment and assesses it with 15 items (23). The questionnaire topics have been formulated in such a way that do not cover professional commitment, as they specifically measure organizational criteria, such as the type of organization and its managers' performance. The Organizational Commitment Scale developed by Allen and Meyer is another instrument that is closer to the professional commitment scale developed in this study in content and has been designed in 3 parts: (1) assessing normative commitment, (2) measuring continuous commitment, (3) and assessing emotional commitment to the organization (24). In management sciences, professional commitment is more applied to the field of organizational commitment. When an individual tries to promote his/her profession, he possesses professional commitment, which may or may not be in line with organizational commitment. In organizational commitment, the individual tends to move in a path that meets the organizations' interests (25), whereas professional commitment is based on the individual's internal tendency to be part of a specific profession apart from the organization.

Another instrument used in different studies to measure commitment to job hierarchies is Blau's Career Commit- ment Scale. Commitment to job hierarchies implies a professional commitment and the individual's attitude toward a profession (26). This scale consists of 7 broad topics that determine the individual's attitude and motivation for being part of a profession and staying in a job, and it applies to any profession.

A review of the literatures reveals the lack of instruments dedicated to measure professional commitment in clinical nurses. Researchers engaged with the concept of professional commitment believe that commitment is a context-dependent phenomenon that is developed within a social context (27). As a result, designing a professional commitment scale for clinical nurses is rather a new area of study and no specific instrument has yet been developed in this field. Therefore, designing a localized, reliable, and valid scale on professional commitment seemed to facilitate future research in this field.

This study will be conducted to design an instrument to measure professional commitment in clinical nurses and examine the validity and reliability of the instrument.

Objectives:

1. Determining the dimensions of the concept of professional commitment in clinical nurses

2. Designing a professional commitment scale for clinical nurses

3. Examining the validity and reliability of the professional commitment scale for clinical nurses

\section{Methods}

The professional commitment scale for clinical nurses will be designed using an exploratory mixed methods approach. The initial objective of this exploratory approach will be to extend the qualitative results obtained from the small study population surveyed in the first step over to the larger study population of the second step of the study. The two-step design of the exploratory approach denotes that the qualitative results obtained in the first step will be able to help the formation of quantitative results in the second step. The exploratory approach is particularly useful when the researchers need to design and test an instrument (28).

The Professional Commitment Scale for clinical nurses will be designed based on Waltz's approach (2010), which involves 4 basic steps: (1) choosing a framework or conceptual model to determine the dimensions of professional commitment in the process of measurement (2) defining the objectives of the measurement, (3) formulating the initial plan or roadmap, (4) and developing the scale through designing the items, setting the methods, and

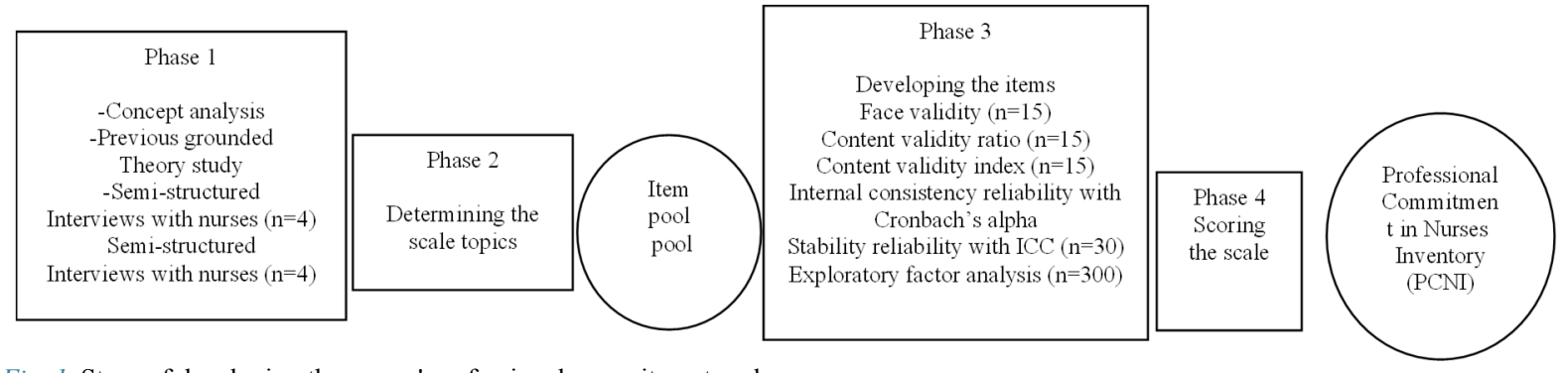

Fig. 1. Steps of developing the nurses' professional commitment scale 
scoring of the scale (29) (Fig. 1). The present study will follow these steps within its 2 major steps, ie, the qualitative step and the quantitative step.

\section{Steps 1 and 2}

Choosing a framework or conceptual model to determine the dimensions of professional commitment and the objectives of the measurement.

Concept analysis: It is an appropriate approach for identifying the dimensions, characteristics, and elements of the concept of professional commitment. The researchers will search databases including CINAHL, MEDLINE, Ovid, and PubMed using the following keywords: "nursing" and "professional commitment". The Google Scholar database will also be used to complete the data collection. The search will be limited to materials published during 2004 and 2016.

Determining the scale topics: This part will be performed through qualitative interviews. The discussed approach gives participants a chance to explain and predict certain events, express a more complete perception of their social life, and create methods for understanding the world around them, and to theoretically express those methods (30).

In the qualitative step of the study, participants will be all the clinical nurses, matrons, and supervisors working at the surveyed hospitals. Individuals with at least one year of experience in clinical services currently working in the nursing profession will be taken as potential participants. Based on the methodology used for qualitative research, participants should be willing to participate in the study and submit their informed written consent prior to entering it, should have adequate power of speech, have some knowledge about professional commitment, and be able to share that knowledge with the interviewer.

The researchers will explain the objectives and methods of the study to participants. After participants agree to being interviewed, the place and time of the interviews will be set in a way that suits the participants. Participants will be selected through maximum variation sampling. Nurses employed at a variety of positions and with different years of service, employment contracts, and places of work can be interviewed. Sampling will continue until data saturation. The number of participants cannot be determined before data saturation; however, given the objectives of the study, the data are expected to be saturated with 20 to 25 participants.

Collecting the qualitative data: In this stage of the study, the data will be collected through in-depth interviews. The interviews will be performed in a quiet place agreed on by both the participant and the interviewer. The maximum duration of each interview will be determined as 2 hours, varying with the conditions. The interviews will be performed by one of the main researchers. To ensure the collection of adequate rich data, the researchers will use the guideline containing all the main interview questions. This guideline consists of an opening section with introductory questions about the personal characteristics of the interviewee, including his/her age, marital status, number of children, years of service, departments of service, and position. Some general questions guiding the interview will then be posed to participants including "Please describe a sample working day in a way that elucidates the concept of professional commitment", "Please explain how you entered the nursing profession and came to accept it", "What are your experiences with nurses' tendency to leave their job?" and "Please describe a situation that shows your highest degree of professional commitment". The answers given by participants will be compared together, and the themes obtained from them will form the basis of the remaining stages of the data collection. All the interviews will be tape recorded with participants' consent.

Analyzing the data and developing the scale topics: In this stage, the data will be analyzed according to qualitative content analysis using a guided approach. The process of analysis involves open coding, classification, and summarization $(31,32)$. Once all the interviews are transcribed, the researchers will merge themselves in the data through reading the texts several times to grasp the general idea of the different aspects of participants' experiences. Sentences will then be converted to meaning units, which are parts of sentences or paragraphs, and the initial codes will thus be obtained using words most closely resembling participants' statements. The codes will then be classified based on their similarities and differences and grouped according to the similarity of their content and its continuity. The categories will then be placed next to one another within a meaningful conceptual model, the relationship between the data will be detected, and the main themes will emerge. MAXQDA-2007 will be used for data analysis. One of the major advantages of this software is its ability to display the main themes and the subthemes in 4 separate parts and on the same page, which enables a quick access to all the texts, codes, categories, and main themes at one glance.

\section{Rigor of the qualitative data}

There is no value to a research without a confirmed rigor of its data, as its findings would not be applicable in real life. Reliability and validity are important aspects of any approach to research that help verify the accuracy of the data (33). In most qualitative studies, reliability and validity are examined based on the 4 criteria set by Lincoln and Guba (1985) including credibility, dependability, confirmability, and transferability ${ }^{(34)}$. The present study will also use these criteria to assess the rigor of its data.

\section{Credibility}

To ensure the credibility of the research data, the researchers will spend a long time collecting the data. Triangulation will be another step the researchers have to take to ensure the credibility of the data obtained. Various sources of data will be used to obtain richer data. Numerous individuals with similar experiences will be interviewed. The entire research team will read the transcribed interviews, the initial codes, the concepts and the themes, and will discuss them in meetings. The initial concepts and themes, the extracted codes, and the developed categories will be presented to some of the study participants for assessment, who will comment on them in meetings 
and make suggestions to the researchers for modifying them. The researchers will then seek to organize their thoughts on each topic and avoid a biased judgment through constraining their thoughts and mentalities throughout the stages of the study including the data collection, data analysis, and theorization stages.

\section{Dependability, confirmability, and transferability}

To ensure the dependability and confirmability of the data, the research will be audited. The researchers will seek to increase the transferability of the data through providing a rich description of participants' characteristics, the sampling method used, and the process of data collection. Expressing parts of participants' statements will also help confirm the extracted codes, themes and subthemes, and improve the transferability of the data.

The analysis of the qualitative data and the extracted themes will lead to the presentation of a framework or conceptual model for determining the dimensions of professional commitment and the objectives of measuring the concept. The target statement for the scale will be formulated, which involves expressing the characteristics of the dimension (the content area) or the construct (the abstract psychological characteristics) that are supposed to be measured, and the characteristics of the target group for which the scale is being designed. The analysis of the qualitative data will determine a variety of potential items that will evaluate the construct as accurately and meaningfully as possible.

Step 3: Formulating the roadmap or developing the scale and designing the items

Developing the items

The qualitative analysis step will lead to the extraction of themes that may be taken as items suitable for measuring professional commitment. All the items and their constituent dimensions will be summed as the "pool of items". To develop a scale that can cover the components of professional commitment in clinical nurses, a review of literature and scales designed on the subject will be added to the examination of the codes extracted from the qualitative step of the study. Moreover, participants' opinions will be used alongside the experts' in the psychometric assessment stage of the study to measure the fit of the items in relation to the scale's constructs. To examine the psychometric properties of the tool, the validity and reliability of the scale will be examined using measures of face, content, and construct validity and reliability assessment.

\section{Face validity}

The qualitative assessment of face validity entails evaluating the apparent relevance of the instrument to what it seeks to measure (35). The quantitative assessment of face validity is performed using the item impact method (36). To conduct a qualitative assessment of the face validity of the scale, it will be distributed among a number of clinical nurses to comment on its level of difficulty, the fit of its items, and their ambiguity. The quantitative assessment of face validity will be performed through measuring the impact of each item after receiving the nurses' ideas about each item's degree of importance to the scale.

\section{Content validity}

Content validity is measured qualitatively and quantitatively. In the qualitative assessment of the content validity of a scale, experts examine the compliance of the scale with the rules of grammar, its use of appropriate words, its proper positioning of the items, and its appropriate scoring system. The content validity index (CVI) and content validity ratio (CVR) are measured to conduct a quantitative assessment of the content validity of a scale. To determine the content validity of the scale designed in the present study, it will be distributed among a group of experts including faculty members of nursing schools and instrument design experts, who will comment on the simplicity, clarity, and appropriate positioning of the items, the scale's scoring system, and the necessity and relevance of each item. An editor will then evaluate the items with respect to their grammar.

CVR: The experts will be requested to comment on the necessity of each item in the scale. The CVR of the scale will then be calculated using an appropriate equation.

CVI: The CVI refers to the experts' agreement on the relevance of each item. The CVI of the scale will be calculated based on the responses given by the experts.

\section{Construct validity}

Determining the factor construct of the scale

The construct validity of the scale designed in the present study will be examined through both exploratory and confirmatory factor analysis methods; a statistical sample of 300 will suffice for these analyses. As in most other studies, the item loading is going to be set as 0.4 .

A pilot test will be performed prior to sampling to examine the construct validity of the scale, and the initial scale will be distributed among representatives of the target group once again. As this sample size has to be large enough to allow quantitative assessment, the initial scale will be distributed among 30 nurses working in clinical wards, who will be requested to conduct a qualitative assessment of the scale once again as they respond to the items and to comment on their clarity and their conformity to the objectives of the scale. The pilot test will provide the data required for determining the reliability of the scale (item analysis). The data obtained will then be statistically analyzed, and Cronbach's alpha values will then be calculated for the initial scale and its subscales.

The version of the scale devised in this step will be an initial instrument with a confirmed face and content validity and will have to be distributed among a larger sample population, so a factor analysis can be performed on its results. The scale will consist of 2 parts. The first part will assess participants' personal details including age, gender, marital status, years of service in nursing, current position, work shift, type of hospital employed at, and status of employment at a second job. Another item will also be added to these details, requiring participants to determine their level of satisfaction with the nursing profession on a scale from $0 \%$ (connoting minimum satisfaction) to $100 \%$ 
(connoting maximum satisfaction). The second part of the scale will assess the dimensions of the items that measure professional commitment in clinical nurses.

In this step, participants will consist of all the clinical nurses, matrons, and nursing managers employed at the surveyed hospitals. Sample size will be calculated using the sample size equation with a $5 \%$ error rate. The pilot study will help the researchers determine a more appropriate sample size while considering any potential sample loss. The researchers determine the study sample size as 300 to obtain appropriate data and prevent potential biases. The inclusion criteria used in this step include having at least one year of work experience as a clinical nurse in hospital departments, or being a hospital supervisor or matron. Participants will be selected through convenience sampling from clinical nurses working at public and private hospitals and hospitals affiliated to different organizations (such as Social Security Hospitals, the Military Hospitals, etc.). A factor analysis will then be performed on the data to examine the construct validity of the scale.

\section{Reliability}

Reliability refers to the repeatability or consistency of scores from one assessment to another. This study will use 3 techniques to ensure the reliability of the scale. The Cronbach's alpha coefficient will first be calculated by collecting the responses of 30 clinical nurses to the initial version of the scale. Allen and Meyer's Organizational Commitment Scale will be distributed among 100 participants as a scale with constructs similar to the constructs formed in the Professional Commitment Scale developed in the present study to assess their concurrent reliability. Allen and Meyer's Organizational Commitment Scale has been used in many studies and thus has a confirmed reliability and validity; it evaluates organizational commitment in 3 areas: (1) continuous commitment, (2) normative commitment, (3) and emotional commitment (24, 37, 38). The concurrent reliability of the scale can thus be examined. The third step to determine the reliability of the scale involves the test-retest method, for which 30 clinical nurses will complete the scale twice with an interval of 2 weeks, and the score obtained for the internal consistency of the items will then be calculated.

\section{Step 4: Scoring the scale}

Given that the scale has not yet been fully developed, the exact scoring of the scale cannot be discussed. However, the data obtained from the scale will be analyzed using descriptive statistics for the quantitative variables including the mean, standard deviation, median, and minimum and maximum values, the number and percentage for the qualitative variables, and inferential statistics to assess the responses. The mean and standard deviation for each group will be measured to assess the correlation between the quantitative variables, while the $t$ test and Pearson's correlation coefficient will be measured to examine the correlation between the score obtained for professional commitment and the demographic variables.

The cross tables, chi square test, and other specific tests will be conducted to examine the correlation between the quantitative variables.

The level of statistical significance will be set at $5 \%$ for all the items $(\alpha=0.05)$.

\section{Ethical considerations}

The research protocol has been approved by the Ethics Committee of Tehran University of Medical Sciences. The researchers consider themselves obliged to be physically present at the research setting after obtaining permission from the relevant authorities and the research deputy of Tehran, Guilan, and Baqiyatallah Universities of Medical Sciences, and the head of the private hospitals selected as the study setting. Participants will be briefed on the methods and objectives of the study and the voluntary nature of participation. All participants will be given a consent form to read and sign. They will then be notified that the interviews are recorded upon their consent and will be ensured that all their data will remain confidential.

Moreover, the participants will be ensured that they can withdraw from the study at any stage and that all the data will remain confidential by keeping participants anonymous and assigning a code to each of them to be used instead of their real names in the publication of the results. The researchers will seek to remain unbiased throughout the entire process of interpreting the qualitative data.

\section{Discussion}

The present study will develop a scale that can measure professional commitment in clinical nurses. This scale will involve clinical nurses' perceptions and statements of professional commitment and will be structured based on their experiences. The scale will also take into an account all the demographic, sociocultural, organizational, and professional characteristics that may have an impact on professional commitment.

\section{Study limitations}

The main limitation of this study will be its subjective nature; however, the researchers will try to disregard their own thoughts and mentalities about the subject under examination throughout the study. The developed scale will be used to measure professional commitment only in clinical nurses and will not apply to other nursing practices.

\section{Conclusion}

The results of this study may help nursing managers better evaluate professional commitment in their clinical nurses. Moreover, this scale can be used in regulatory and macro managing systems to conduct further evaluations and resolve the existing barriers in professional commitment of nurses.

\section{Acknowledgement}

The researchers would like to express their gratitude to all the nurses participating in the interviews and the nurses completing the scales. The project has been funded by the Nursing and Midwifery Care Research Center, Tehran University of Medical Sciences. 
Conflict of Interests

The authors declare that they have no competing interests.

\section{References}

1. McKechnie T. Call for nurses: The Multi-Dimensional Manifestation of Nursing Shortage on Patient Care. QIHI Journal of Healthcare Improvement and Patient Safety 2016(2).

2.Zhu J, Rodgers S, Melia KM. A qualitative exploration of nurses leaving nursing practice in China. Nurs Open. 2015;2(1):3-13.

3. Rudman A, Gustavsson P, Hultell D. A prospective study of nurses' intentions to leave the profession during their first five years of practice in Sweden. Int J Nurs Stud. 2014;51(4):612-24.

4. Gould D, Fontenla M. Commitment to nursing: results of a qualitative interview study. J Nurs Manag 2006; 14(3):213-21.

5. Zhu J, Rodgers S, Melia KM. Can Education Resolve Nursing Shortage in China? Athens Journal of Health. 2015.

6. Rafii F, Hajinezhad ME, Haghani $\mathrm{H}$. Nurse caring in Iran and its relationship with patient satisfaction. Aust J Adv Nurs. 2008;26(2):75.

7. Mirzabeigi G, Salemi S, Sanjari M, Shirazi F, Heidari S, Maleki S. Job Satisfaction among Iranian Nurses. Hayat. 2009;15(1).

8. Nehrir B, Ebadi A, Sh T, AA KZ, Honarvar H. Relationship of job satisfaction and organizational commitment in hospital nurses. Journal Mil Med. 2010;12(1):23-6.

9. Vanaki Z, Vagharseyyedin SA. Organizational commitment, work environment conditions, and life satisfaction among Iranian nurses. Nurs Health Sci. 2009;11(4):404-9.

10. Jahangir F, Shokrpour N. Three components of organizational commitment and job satisfaction of hospital nurses in Iran. Health Care Manag. 2009;28(4):375-80.

11. Ramoo V, Abdullah KL, Piaw CY. The relationship between job satisfaction and intention to leave current employment among registered nurses in a teaching hospital. J Clin Nurs. 2013;22(2122):3141-52.

12. Kuokkanen L, Leino-Kilpi H, Katajisto J. Nurse empowerment, jobrelated satisfaction, and organizational commitment. J Nurs Care Qual. 2003;18(3):184-92.

13. Gess E, Manojlovich M, Warner S. An evidence-based protocol for nurse retention. J Nurs Adm. 2008;38(10):441-7.

14. McCabe T, Sambrook S. Psychological contracts and commitment amongst nurses and nurse managers: A discourse analysis. Int J Nurs Stud. 2013;50(7):954-67.

15. McCabe TJ, Garavan TN. A study of the drivers of commitment amongst nurses: The salience of training, development and career issues. J Europ Indust Train. 2008;32(7):528-68.

16. Jafaraghaee $\mathrm{F}$, Mehrdad N, Parvizy S. Influencing factors on professional commitment in Iranian nurses: A qualitative study. Iran J Nurs Midwifery Res. 2014;19(3):301.

17. Jafaraghaie F, Parvizy S, Mehdad N, Rafii F. Concept analysis of professional commitment in Iranian nurses. Iran J Nurs Midwifery Res. 2013;17(7).

18. Akbari O, Vagharseyyedin SA, Farajzadeh Z. The Relationship of organizational justice with nurses' professional commitment. Mod Care J. 2015;12(1):31-8.

19. Jiang Z. Emotional Intelligence and Career Decision-Making SelfEfficacy: Mediating Roles of Goal Commitment and Professional Commitment. J Employ Couns. 2016;53(1):30-47.

20. Kathleen Anne Blakely Duncan KAB. Behavioral indicators of staff nurses' organizational and professional commitment" (January 1, 1994). ETD collection for University of Nebraska - Lincoln. PaperAAI9504140. http://digitalcommons.unl.edu/dissertations/AAI9 504140

21. Jones JMS. The impact of hospital mergers on organizational culture, organizational commitment, professional commitment, job satisfaction and intent to turnover on registered professional nurses on medicalsurgical hospital units (Doctoral Dissertation Research). State University of New York, Buffalo; 2000.

22. Wolf ZR, Hoerst B. Professional commitment in RN-BSN and basic BSN students: program evaluation. Nurse Educ. 2007;32(2):61-5.

23. Kanning UP, Hill A. Validation of the Organizational Commitment Questionnaire (OCQ) in six languages. JBMP. 2013;4(2):11-20.

24. Jaros S. Meyer and Allen model of organizational commitment: Measurement issues. The Icfai J Organiz Behav. 2007;6(4):7-25.

25. LLapa-Rodríguez EO, Trevizan MA, Shinyashiki GT. Conceptual reflections about organizational and professional commitment in the health sector. RLAE. 2008;16(3):484-8.

26. Blau G. Testing for a four-dimensional structure of occupational commitment. J Occup Organ Psychol. 2003;76(4):469-88.

27. Cohen A. Commitment before and after: An evaluation and reconceptualization of organizational commitment. HRMR. 2007;17(3):336-54.

28. Creswell JW, Klassen AC, Plano Clark VL, Smith KC. Best practices for mixed methods research in the health sciences. Bethesda (Maryland): National Institutes of Health. 2011:2094-103.

29. Waltz C, Stickland O, Lenz E. Nursing and Health Research. Sage, Newbury Park, CA; 2010.

30. De-La-Cueva-Ariza L, Romero-García M, Delgado-Hito P, AcostaMejuto B, Jover-Sancho C, Ricart-Basagaña M, et al. Development of an instrument to measure the degree of critical patient's satisfaction with nursing care: research protocol. J Advnursing. 2014;70(1):20110.

31. Elo S, Kyngäs $\mathrm{H}$. The qualitative content analysis process. J Advanced Nurs. 2008;62(1):107-15.

32. Hsieh HF, Shannon SE. Three approaches to qualitative content analysis. Qual Health Res. 2005;15(9):1277-88.

33. Morse JM, Barrett M, Mayan M, Olson K, Spiers J. Verification strategies for establishing reliability and validity in qualitative research. IJQM. 2002;1(2):13-22.

34. Polit D, Beck C. Nursing research: Generating and assessing evidence for practice. Philadelphia, PA: Lippincott Williams \& Wilkins; 2008.

35. Houser J. Nursing research: Reading, using and creating evidence: Jones \& Bartlett Publishers; 2013.

36. Broder HL, McGrath C, Cisneros GJ. Questionnaire development: face validity and item impact testing of the Child Oral Health Impact Profile. Community Dent Oral Epidemiol. 2007;35(s1):8-19.

37. Meyer JP, Stanley DJ, Herscovitch L, Topolnytsky L. Affective, continuance, and normative commitment to the organization: A metaanalysis of antecedents, correlates, and consequences. J Vocat Behav. 2002;61(1):20-52.

38. Lee K, Allen NJ, Meyer JP, Rhee KY. The Three-Component Model of Organisational Commitment: An Application to South Korea. Applied Psychology. 2001;50(4):596-614 